\title{
Successful Management of Pulmonary Arterial Hypertension by Monitoring N-Terminal Pro-B-Type Natriuretic Peptide Serum Levels in a Preterm Infant with Chronic Lung Disease: A Case Report
}

\author{
Keigo Seki, MD ${ }^{1}$ Satoru Iwashima, $\mathrm{PhD}^{1}$ Hiroki Uchiyama, $\mathrm{MD}^{1}$ Akira Ohishi, $\mathrm{PhD}^{1}$ \\ Takamichi Ishikawa, $\mathrm{PhD}^{1}$ \\ ${ }^{1}$ Department of Pediatrics, Hamamatsu University School of \\ Medicine, Hamamatsu, Japan \\ Address for correspondence Satoru Iwashima, MD, Department of \\ Pediatrics, Chutoen General Medical Center, Syoubugauraike 1-1, \\ Kakegawa City, 436-8555, Japan (e-mail: iwashima3617@gmail.com).
}

Am J Perinatol Rep 2019;9:e133-e137.

\author{
Abstract \\ Keywords \\ - NT-proBNP \\ - bronchopulmonary \\ dysplasia \\ - chronic lung disease \\ - pulmonary arterial \\ hypertension \\ - tracheobron- \\ chomalacia
}

We measured the serial changes in N-terminal probrain natriuretic peptide (NT-proBNP) levels in a 6-month-old male infant with chronic lung disease (CLD) complicated by pulmonary arterial hypertension (PAH). The patient was born at the 24th week of gestation weighing $695 \mathrm{~g}$. At 1 month after birth, an echocardiogram confirmed the diagnosis of CLD with PAH. He was treated with inhaled nitric oxide (iNO) and oral sildenafil and discharged from the hospital. At 190 days of age, the patient was readmitted to our department because of a viral upper respiratory infection. At 195 days of age, his respiratory condition worsened with pulmonary edema and his NT-proBNP level was determined to be $10,117 \mathrm{pg} / \mathrm{mL}$. The patient was immediately administered iNO, and his respiratory condition improved, and NT-proBNP levels decreased. However, he experienced repeated severe cyanosis attacks. Before the attacks, his NT-proBNP level was $>1,000 \mathrm{pg} / \mathrm{mL}$. Therefore, we continuously administered iNO until his NT-proBNP level decreased to $<1,000 \mathrm{pg} / \mathrm{mL}$. We safely discontinued iNO administration at 473 days of age. In conclusion, serial change in NT-proBNP is a surrogate marker with prognostic value in patients with PAH associated with CLD.

Pulmonary arterial hypertension (PAH) complicates the course of chronic lung disease (CLD) in newborns and contributes to later morbidity and mortality during infancy, especially in the context of bronchopulmonary dysplasia (BPD). Earlier studies showed that patients with CLD and later-onset PAH represent a very high-risk population that exhibits increased morbidity and mortality. ${ }^{1}$ Among infants with BPD, 20 to $40 \%$ develop $\mathrm{PAH}$ at some point during their initial hospital course, and PAH is an important risk factor for mortality in this population. 2,3 Echocardiography is often used both to screen for and monitor the progression of PAH in infants with BPD. However, it has been reported that echocardiographic screening at 28 days of life fails to identify many late-developing cases of PAH in

received

November 5, 2018 accepted after revision February 24, 2019
DOI https://doi.org/

10.1055/s-0039-1684026. ISSN 2157-6998. extremely low-birth-weight infants. ${ }^{4}$ There are many causes for failed or late diagnosis of PAH in CLD, such as poor imaging due to over-inflation caused by CLD, the complexities of evaluating PAH by echocardiography, and dramatic changes in the hemodynamic status of PAH in CLD due to the patient's unstable respiratory condition. Recently, N-terminal probrain natriuretic peptide (NT-proBNP) levels and BNP levels have been useful in screening for PAH in preterm infants with CLD who are at risk for death. ${ }^{5,6}$ However, few studies have elucidated the changes in NT-proBNP levels over time in CLD complicated by $\mathrm{PAH}$. In this report, we determined the serial changes in NT-proBNP levels in a 6-month-old male infant with CLD complicated by PAH.
Copyright $\odot 2019$ by Thieme Medical Publishers, Inc., 333 Seventh Avenue, New York, NY 10001, USA. Tel: +1(212) 584-4662.
License terms

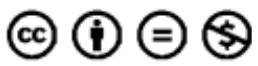




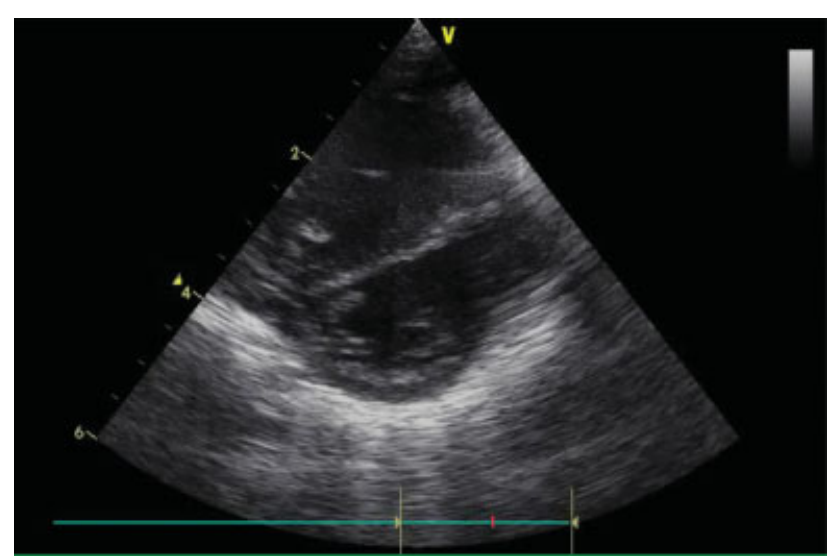

Fig. 1 Echocardiography (aortic valve short axis image). Observation of flattening of the ventricular septum.

\section{Patient Report}

The patient's mother was referred to our department for premature rupture of the membranes at the 24th week of gestation. An emergency cesarean section was required because of oligohydramnios and indications of fetal distress. The patient's birth weight was $695 \mathrm{~g}$ and his Apgar's scores were 3 and 7 at 1 and 5 minutes, respectively. The patient was immediately intubated with surfactant and admitted to our newborn intensive care unit. His respiratory condition was poor and he could not be weaned from mechanical ventilator support. One month after birth, an X-ray examination was performed, and the patient was diagnosed with CLD. His oxygenation level was poor. An echocardiogram revealed no congenital heart disease, but PAH was observed with right atrial enlargement, right ventricular dilation, and septal flattening ( $\mathbf{- F i g . ~ 1 ) . ~ H e ~ w a s ~ t r e a t e d ~ w i t h ~ i n h a l e d ~ n i t r i c ~ o x i d e ~}$ (iNO), oral sildenafil, diuretics, and intravenous and inhaled steroids. Progressive improvement in his respiratory condition led to subsequent extubation and, at 169 days of age, his weight had increased to 2,568 g. At this time, the patient was discharged and prescribed supplemental oxygen $(1.0 \mathrm{~L} / \mathrm{min}$ by nasal cannula). At 190 days of age, he was readmitted to our department due to a viral upper respiratory infection. At 195 days of age, his respiratory condition and oxygenation level worsened and he developed pulmonary edema. His peripheral capillary oxygen saturation $\left(\mathrm{SpO}_{2}\right)$ was $67 \%$ and he developed cardiopulmonary arrest. We immediately performed successful cardiopulmonary resuscitation. His clinical course is shown in - Fig. 2. His laboratory findings were: white

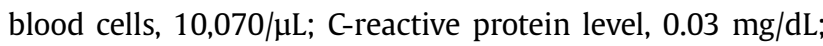

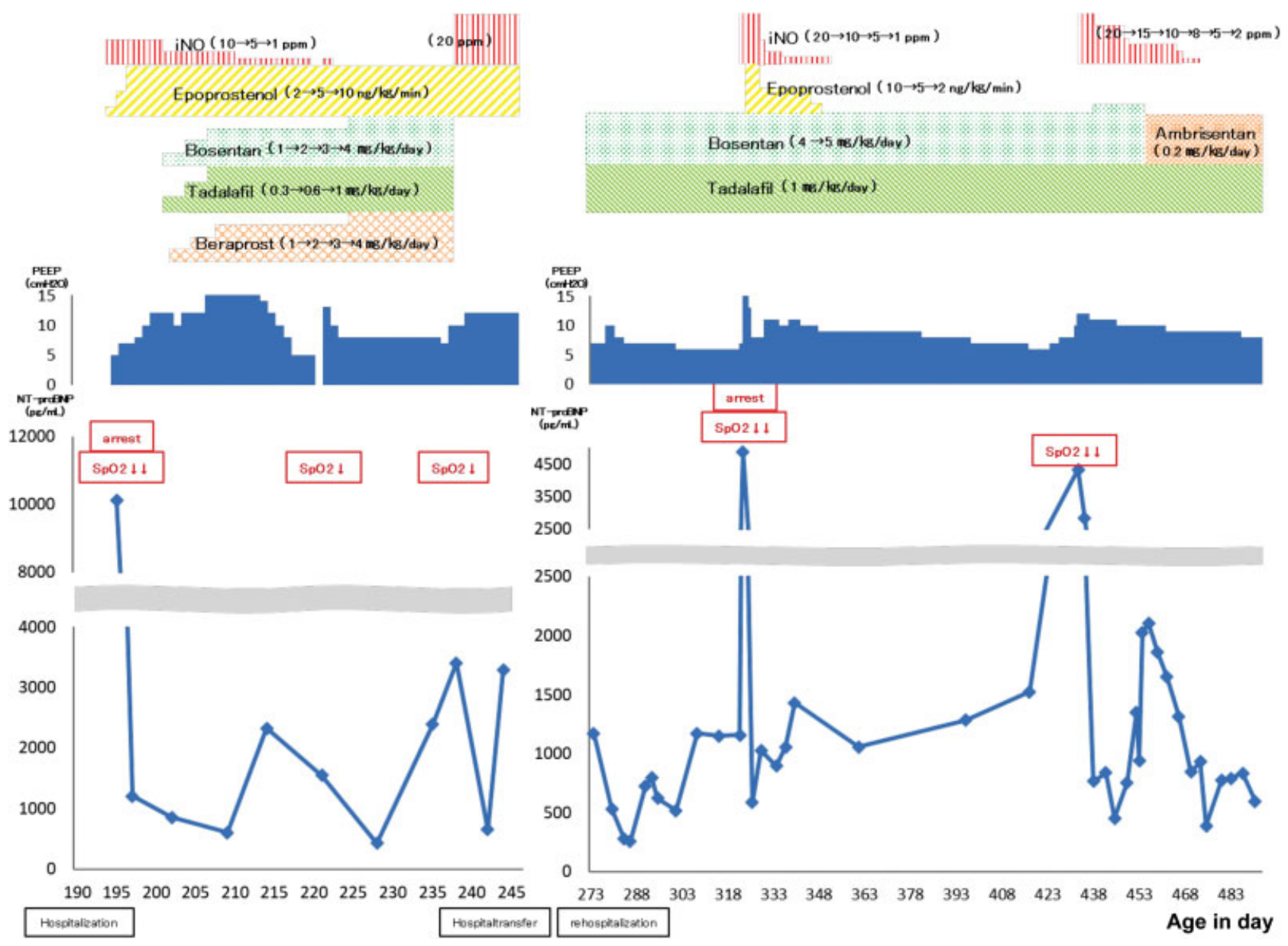

Fig. 2 Serial change in NT-proBNP level and the patient's clinical course. Over the course of treatment, there were six instances during which the NT-proBNP levels increased, of which three instances were associated with severe cyanosis attacks. iNO, inhaled nitric oxide; NT-proBNP, $\mathrm{N}$-terminal pro-B-type natriuretic peptide; $\mathrm{SpO}_{2}$, oxygen saturation of peripheral artery. 
and NT-proBNP level, $10,117 \mathrm{pg} / \mathrm{mL}$, as indicated by an Elecsys 2010 analyzer with a chemiluminescent immunoassay kit (Roche Diagnostics, Mannheim, Germany). The protocol for this assay has been previously described. ${ }^{7}$ We could not determine the NT-proBNP levels during his stay in the neonatal intensive care unit because echocardiography performed after he was treated with iNO, oral sildenafil, and diuretics showed improvement of the characteristic PAH signs (right atrial enlargement, right ventricular dilation, and septal flattening). After his condition stabilized, we continued the administration of iNO, catecholamines, and epoprostenol and measured his NT-proBNP levels two to three times per week. His $\mathrm{SpO}_{2}$ level increased and his respiratory condition improved after treatment, with the levels of NT-proBNP decreasing to $1,201 \mathrm{pg} / \mathrm{mL}$ at 197 days of age. However, echocardiography indicated that his PAH did not improve; therefore, we added oral bosentan and tadalafil to his treatment regimen and, at 201 days of age, we were able to discontinue the iNO. We were not able to wean the patient from mechanical ventilator support and we transported him to another institution to perform a tracheotomy while maintaining oral bosentan and tadalafil. During his stay at the other institution, there were no episodes of pulmonary hypertension. After the tracheotomy was performed, we performed a bronchoscopy and confirmed a diagnosis of tracheobronchomalacia. High positive-end expiratory pressure (PEEP) therapy was started for the tracheobronchomalacia and the patient's respiratory condition was improved. We then started to wean him from mechanical ventilator support. At the age of approximately 300 days, when the PEEP setting was decreased from 7 to $6 \mathrm{~mm} \mathrm{Hg}$, the patient's NT-proBNP level slightly increased to $1,171 \mathrm{pg} / \mathrm{mL}$ at 308 days of age.

However, at 323 days of age, he suddenly experienced a severe cyanosis attack (after crying), and the suspected cause was PAH or uncontrolled tracheobronchomalacia. Cardiopulmonary resuscitation was initiated and iNO, catecholamines, and epoprostenol were administered. High PEEP therapy was performed for tracheobronchomalacia which was difficult to control. Furthermore, the patient experienced repeated severe cyanosis attacks at 427 days of age after ending iNO therapy. His NT-proBNP levels were slightly increased and were always $>1,000 \mathrm{pg} / \mathrm{mL}$ when tracheobronchomalacia was hard to manage and he exhibited unstable respiratory conditions. We continued to administer iNO even when the NT-proBNP values were $<1,000 \mathrm{pg} / \mathrm{mL}$. At 454 days of age, the patient's NT-proBNP value had slightly increased and we suspected the re-emergence of an unstable respiratory condition due to tracheobronchomalacia. After we maintained $10 \mathrm{~mm} \mathrm{Hg}$ PEEP, his NT-proBNP level decreased. He was subsequently weaned from iNO therapy and his medication was changed from bosentan to ambrisentan. Subsequently, his NT-proBNP values decreased to $<1,000 \mathrm{pg} / \mathrm{mL}$ and iNO therapy was stopped at 473 days of age. His respiratory condition, which was caused by tracheobronchomalacia, gradually stabilized and high PEEP therapy was reduced. When the patient was 2 years and 2 months of age, we performed cardiac catheterization. His mean pulmonary arterial pressure was $24 \mathrm{~mm} \mathrm{Hg}$ and, under treatment with tadalafil and ambrisentan, there was no evidence of PAH. Furthermore, at 2 years and 4 months of age, his NT-proBNP value was $350 \mathrm{pg} / \mathrm{mL}$. He was discharged with a home respiratory machine, supplemental oxygen, and ongoing treatment with tadalafil and ambrisentan. After discharge, these therapies will be carefully tapered since infants with CLD have increased risks of mortality due to respiratory failure, unremitting pulmonary hypertension with cor pulmonale, or acquired infection (pneumonia or sepsis).

\section{Discussion}

We determined the serial changes in NT-proBNP levels in a 6month-old male infant with CLD complicated by PAH retrospectively. Our patient experienced five cyanotic attacks, including two requiring CPA. Before his severe cyanotic attacks developed, the NT-proBNP levels were nearly always slightly increased to $>1,000 \mathrm{pg} / \mathrm{mL}$. The elevated NT-proBNP was a cause of one CPA, while the others might have been due to the $\mathrm{PAH}$. When we failed to wean him off the iNO therapy for PAH twice, his NT-proBNP levels were unstable and exceeded 1,000 $\mathrm{pg} / \mathrm{mL}$. Hence, we decided to maintain the iNO therapy until the NT-proBNP dropped to less than $1000 \mathrm{pg} / \mathrm{mL}$ after which the iNO was successfully stopped.

NT-proBNP is the precursor of brain natriuretic peptide. This peptide is released from the myocardial tissue of the right and left ventricles when stretched. NT-proBNP is helpful in diagnosing heart failure; however, it does not distinguish between left or right ventricular failure. In the present case, NT-proBNP levels increased with hemodynamic changes associated with $\mathrm{PAH}$ and respiratory instability. NT-proBNP and BNP are two different types of peptides but, as reported previously, there is a strong correlation between NT-proBNP and BNP levels during childhood. ${ }^{8}$ It is also possible that the measurement results of these peptides reflect the time lag between the time of specimen collection and measurement. Since NT-proBNP has a longer half-life (70 minutes) than atrial NP (12 minutes) or BNP (15 minutes), ${ }^{9}$ it is likely less influenced by stress on the heart just prior to blood collection. In adults, NT-proBNP correlates with right ventricle function in PAH and has been shown to be of prognostic value in $\mathrm{PAH} .{ }^{10,11}$ In the present case, the serial changes in NT-proBNP levels were observed to correlate with the patient's respiratory condition and the hemodynamic changes associated with PAH. While no optimal cut-off value of NT-proBNP, such as $1,000 \mathrm{pg} / \mathrm{mL}$, has been determined, Cuna et al previously reported that a BNP value of $>220 \mathrm{pg} / \mathrm{mL}$ might serve as a prognostic marker of all-cause mortality in extremely low-birth-weight infants with BPD-associated PAH. ${ }^{12}$ Sugimoto et $\mathrm{al}^{8}$ reported a strong correlation between BNP and NT-proBNP levels, where NT-proBNP $=9.080 \times \mathrm{BNP}^{0.923}$. We speculated that at a threshold of approximately $2000 \mathrm{pg} / \mathrm{mL}$, NT-proBNP might serve as a prognostic indicator, and that our patients level $(<1000 \mathrm{pg} / \mathrm{dL})$ might be a safe level at which to control the CLD complicated by PAH. We speculated that the serial changes in NT-proBNP levels observed in this case were associated with the patient's PAH with CLD. Furthermore, high levels of NT-proBNP might be useful in predicting the risk of severe cyanotic attacks. 
Echocardiography is often used both to screen for and to monitor the progression of PAH in infants with CLD. Right ventricular structural or functional abnormalities, as determined by an echocardiogram, are used as indirect indicators of PAH, including septal flattening, right atrial enlargement, right ventricular hypertrophy, dilation, and decreased function. Certain quantitative echocardiographic parameters used to screen for PAH, such as tricuspid annular plane systolic excursion, tricuspid regurgitant jet velocity, and/or pulmonary systolic time intervals, are supported by the 2015 guidelines from the American Heart Association. ${ }^{13}$ However, these parameters have not been validated in preterm infants. There are several causes for failed or late diagnosis of $\mathrm{PH}$ in CLD, such as poor electrocardiogram quality due to CLD-induced over-inflation, as in the present case. While an echocardiogram obtained upon admission revealed severe $\mathrm{PAH}$, as indicated by right atrial enlargement, right ventricular dilation, and septal flattening, we were unable to confirm PAH using echocardiography due to the unstable respiratory conditions and high PEEP therapy. Recently, consensus recommendations for the care of children with bronchopulmonary dysplasia- $\mathrm{PAH}^{14}$ were published. Serial NT-proBNP levels may be useful in monitoring disease progression/regression and response to therapy and in modulating decision making, but these biomarkers should be used in conjunction with echocardiography rather than in isolation. We suggest that NT-proBNP level may be a useful biochemical marker in predicting the presence of PAH in CLD infants.

Tracheobronchomalacia is an acquired complication of BPD and is more common in infants and children with classic BPD who are treated with prolonged positive pressure ventilation. Tracheobronchomalacia is characterized by abnormally compliant, collapsible central airways and may be associated with barotrauma, chronic or recurrent infection, chronic aspiration, and endotracheal intubation. Tracheobronchomalacia can improve with age, as the tracheal cartilage matures and becomes less compliant. ${ }^{15}$ Our patient was diagnosed with tracheobronchomalacia after undergoing a tracheotomy. After this diagnosis, he experienced at least two severe cyanotic attacks induced by $\mathrm{PAH}$. Interestingly, tracheobronchomalacia is easily inducible by $\mathrm{PAH}$, which requires long-term control by a respiratory machine that in turn, sometimes induces unstable respiratory conditions, such as desaturation, hypercapnia, and apneic attack.

\section{Conclusion}

In conclusion, serial changes in NT-proBNP levels can serve as a surrogate marker in place of an electrocardiogram. These changes have been shown to have good prognostic value in patients with PAH associated with CLD. Patients who exhibit higher and unstable levels of NT-proBNP might be developing a serious respiratory and hemodynamic condition. As a result, PAH with CLD should be evaluated carefully and the therapeutic strategy used should be mindful of potential complications, such as tracheobronchomalacia.

\section{Funding}

Equipment provided by the Hamamatsu University School of Medicine was used. This research received no specific grant from any funding agency in the public, commercial, or not-for-profit sectors. Each author listed on the manuscript has seen and approved the submission of this version of the manuscript and takes full responsibility for the manuscript. If the article is accepted for publication, the disclosure statement may be published. This study has no sponsor. This is a case report. The decision to submit the manuscript for publication was made by Satoru Iwashima. The author who wrote the first draft of the manuscript is Satoru Iwashima. There was no honorarium, grant, or other form of payment made to produce this manuscript.

\section{Conflicts of Interest}

The authors of this manuscript do not have any potential, perceived, or real conflicts of interest, including any financial arrangement to disclose.

\section{Acknowledgments}

The authors thank Shigeo Iijima and for their valuable assistance in the treatment of the patients admitted to the Maternal-Fetal and Neonatal Care Center or the Department of Pediatrics at Hamamatsu University School of Medicine.

\section{References}

1 Khemani E, McElhinney DB, Rhein L, et al. Pulmonary artery hypertension in formerly premature infants with bronchopulmonary dysplasia: clinical features and outcomes in the surfactant era. Pediatrics 2007;120(06):1260-1269

2 Slaughter JL, Pakrashi T, Jones DE, South AP, Shah TA. Echocardiographic detection of pulmonary hypertension in extremely low birth weight infants with bronchopulmonary dysplasia requiring prolonged positive pressure ventilation. J Perinatol 2011;31(10): 635-640

3 Check J, Gotteiner N, Liu X, et al. Fetal growth restriction and pulmonary hypertension in premature infants with bronchopulmonary dysplasia. J Perinatol 2013;33(07):553-557

4 Bhat R, Salas AA, Foster C, Carlo WA, Ambalavanan N. Prospective analysis of pulmonary hypertension in extremely low birth weight infants. Pediatrics 2012;129(03):e682-e689

5 Kalra VK, Aggarwal S, Arora P, Natarajan G. B-type natriuretic peptide levels in preterm neonates with bronchopulmonary dysplasia: a marker of severity? Pediatr Pulmonol 2014;49(11): 1106-1111

6 Joseph L, Nir A, Hammerman C, Goldberg S, Ben Shalom E, Picard E. N-terminal pro-B-type natriuretic peptide as a marker of bronchopulmonary dysplasia in premature infants. Am J Perinatol 2010;27(05):381-386

7 Collinson PO, Barnes SC, Gaze DC, Galasko G, Lahiri A, Senior R. Analytical performance of the $\mathrm{N}$ terminal pro $\mathrm{B}$ type natriuretic peptide (NT-proBNP) assay on the Elecsys 1010 and 2010 analysers. Eur J Heart Fail 2004;6(03):365-368

8 Sugimoto M, Manabe H, Nakau K, et al. The role of N-terminal proB-type natriuretic peptide in the diagnosis of congestive heart failure in children. - Correlation with the heart failure score and comparison with B-type natriuretic peptide -. Circ J 2010;74(05): 998-1005 
9 Pemberton CJ, Johnson ML, Yandle TG, Espiner EA. Deconvolution analysis of cardiac natriuretic peptides during acute volume overload. Hypertension 2000;36(03):355-359

10 Leuchte HH, Baumgartner RA, Nounou ME, et al. Brain natriuretic peptide is a prognostic parameter in chronic lung disease. Am J Respir Crit Care Med 2006;173(07):744-750

11 Fijalkowska A, Kurzyna M, Torbicki A, et al. Serum N-terminal brain natriuretic peptide as a prognostic parameter in patients with pulmonary hypertension. Chest 2006;129(05):1313-1321

12 Cuna A, Kandasamy J, Sims B. B-type natriuretic peptide and mortality in extremely low birth weight infants with pulmonary hypertension: a retrospective cohort analysis. BMC Pediatr 2014;14:68

13 Abman SH, Hansmann G, Archer SL, et al; American Heart Association Council on Cardiopulmonary, Critical Care, Periopera- tive and Resuscitation; Council on Clinical Cardiology; Council on Cardiovascular Disease in the Young; Council on Cardiovascular Radiology and Intervention; Council on Cardiovascular Surgery and Anesthesia; and the American Thoracic Society. Pediatric pulmonary hypertension: guidelines from the American Heart Association and American Thoracic Society. Circulation 2015;132 (21):2037-2099

14 Krishnan U, Feinstein JA, Adatia I, et al; Pediatric Pulmonary Hypertension Network (PPHNet). Evaluation and management of pulmonary hypertension in children with bronchopulmonary dysplasia. J Pediatr 2017;188:24-34.e1

15 Shaffer TH, Bhutani VK, Wolfson MR, Penn RB, Tran NN. In vivo mechanical properties of the developing airway. Pediatr Res 1989;25(02):143-146 